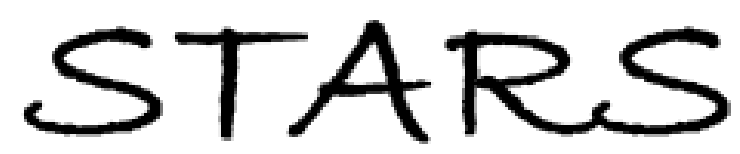

University of Central Florida

STARS

Faculty Scholarship and Creative Works

$6-2015$

\title{
Individual Differences in Digital Badging: Do Learner Characteristics Matter?
}

Joey R. Fanfarelli

University of Central Florida, joey@ucf.edu

Thomas Rudy McDaniel

University of Central Florida

Part of the Adult and Continuing Education Commons, Educational Assessment, Evaluation, and Research Commons, Educational Methods Commons, Educational Psychology Commons, Game Design Commons, Instructional Media Design Commons, Online and Distance Education Commons, and the Scholarship of Teaching and Learning Commons

Find similar works at: https://stars.library.ucf.edu/ucfscholar University of Central Florida Libraries http://library.ucf.edu

This Paper is brought to you for free and open access by STARS. It has been accepted for inclusion in Faculty Scholarship and Creative Works by an authorized administrator of STARS. For more information, please contact STARS@ucf.edu.

\section{STARS Citation}

Fanfarelli, Joey R. and McDaniel, Thomas Rudy, "Individual Differences in Digital Badging: Do Learner Characteristics Matter?" (2015). Faculty Scholarship and Creative Works. 108.

https://stars.library.ucf.edu/ucfscholar/108

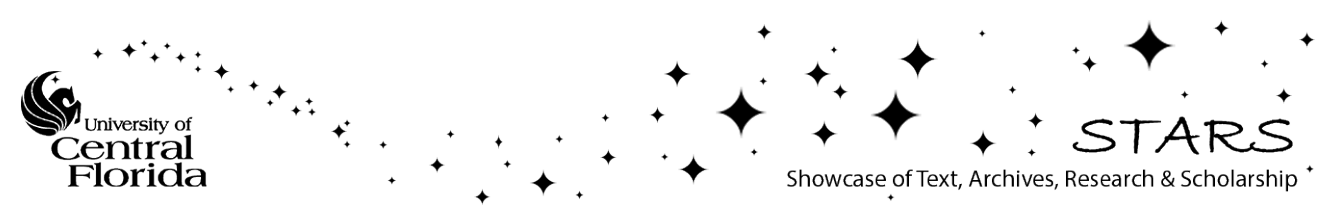




\title{
INDIVIDUAL DIFFERENCES IN DIGITAL BADGING: DO LEARNER CHARACTERISTICS MATTER?
}

\author{
Joseph R. Fanfarelli \\ Rudy McDaniel \\ University of Central Florida
}

\begin{abstract}
Badge use has rapidly expanded in recent years and has benefited a variety of applications. However, a large portion of the research has applied a binary useful or not useful approach to badging. Few studies examine the characteristics of the user and the impact of those characteristics on the effectiveness of the badging system. This study takes preliminary steps toward that cause, examining the effectiveness of a badging system across two web-based university courses in relation to the individual differences of the learners. Individual differences are examined through the lens of Long-Dziuban reactive behavior types and traits. Results revealed differences in badge effectiveness that were dependent upon students' Long-Dziuban categorization. Student engagement, intrinsic motivation, reflective \& integrative learning, and higher order learning were the constructs most dependent upon categorization. Additional results and their implications are discussed within.
\end{abstract}




\section{INTRODUCTION}

In response to the proliferation of distance learning and web-based academia, educators have sought complementary technologies. One such technology, digital badging, is accelerating to the forefront of pedagogical interest (Carey, 2012; Khaddage, Baker, \& Knezek, 2012; Rosewell, 2012; Mehta et al., 2013). Digital badging systems promise increased granularity of assessment (Abramovich, Schunn, \& Higashi 2013), elevated time on task (Blair 2012), and more precise markers of academic success (Rosewell, 2012).

A digital badge can be considered a visual marker of achievement that is awarded in response to the completion of pre-specified criteria and exists in a virtual space (Frederiksen, 2013). For the uninitiated, the digital badge can best be considered in relation to its forebearers. Like military ribbons and Boy and Girl Scout merit badges, digital badges represent what the earner has achieved.

Many of the benefits of digital badging systems are steadily gaining the attention of educators and online systems designers. Consequently, badging has undergone rapid implementation. For example, Mozilla, creators of the Firefox web browser, have created the OpenBadges system, a non-proprietary interface that displays qualification-based badges issued by any organization that wishes to participate (Mozilla, 2014). Several universities have also begun incorporating badging systems, such as Purdue (Purdue, 2014) and UC-Davis (Steward, 2013).

The large-scale adoption of such a technology necessitates the discovery of optimized methods of implementation. It is not enough to simply know that badges seem to improve pedagogy; A deep understanding of how they improve, why they improve, and for whom they improve facilitates the adoption of such a system in a useful way.

Badging has primarily been treated as a one size fits all possibility - Do badges globally improve motivation? Do badges globally alter performance? The answers may be more complex than the questions. Perhaps it is the case that badges do improve motivation - sometimes, for some learners; but not always, for all learners. Research has begun examining the design aspects of badging systems, but, with some exceptions (Abramovich, Schunn, \& Higashi 2013; Hakulinen \& Auvinen 2014), learner characteristics have largely been ignored. If the qualities of the learner impact the effectiveness of the system, implementation 
may require alteration, using badging systems for students who benefit most and omitting them where they discourage the desired results. This study seeks to advance the knowledge-base on individual differences as they relate to badging systems, with a specific focus on reactive behavior types and traits (Long, 1975; 1985).

\section{BACKGROUND}

\section{Badging Effectiveness}

The complete benefits of badges are still being established, but several studies have already revealed positive results. In one large-scale experiment, badges significantly increased student contributions and time on task (Denny, 2013). Blair (2012) tested the effects of badges on participants playing a video game, and showed that well-designed achievements can improve motivation. Fitz-Walter et al. (2011) found that badges enhanced motivation in a cell-phone app meant to orient new students on campus. Additionally, Charleer et al. (2013) showed that badges improved awareness of the requirements for successful completion of a Human Computer Interaction course.

\section{Individual Differences}

While badging systems are proving beneficial in many instances, initial research shows that different learners seem to have differing experiences when exposed to badging systems. Abramovich, Schunn, and Higashi (2013) found evidence that the amount and direction of student motivational changes, after the introduction of a badging system, differed in relation to student pre-ability in math. Hakulinen and Auvinen (2014) discovered differences in motivation to obtain badges between students of different goal orientations. While badging was highly effective for some, it did not appear to exhibit universal appeal. Unfortunately, a shortage of research in this domain leaves questions about the prevalence of such differences as well as the size of their impacts. 


\section{Reactive Behavior Types}

Reactive behavior types and traits (Dziuban, Moskal, \& Dziuban, 2000) present an interesting framework for the study of individual differences in an educational system. According to Long $(1975 ; 1985)$, students tend to behave in accordance with one of four reactive behavior types:

1. Aggressive Independent - High in energy with little need for approval, they prefer to work alone and are frequently disorganized and impulsive. Direct with others, they prefer to solve situations in real-time, not proactively.

2. Passive Independent - Low in energy with little need for approval, they prefer not to participate and may act contrarily to their own best interests. Frequently underachieving, they may develop negative feelings toward personal academic ability.

3. Aggressive Dependent - High in energy with high need for approval, they are motivated to participate and actively seek help outside of class.

Though frequently high achievers, peer esteem increases stress instead of satisfaction.

4. Passive Dependent - Low in energy with high need for approval, they are compliant and non-confrontational. Gentle and caring, their need for approval causes disagreement and criticism to be interpreted as personal rejection.

These types may also be considered in terms of aggressive types vs passive types or independent types vs dependent types. Energy levels are denoted by aggressive (high) and passive (low), while need for approval is denoted by dependent (high) and independent (low).

Behavior type may be associated with zero to four supporting traits (Dziuban, Moskal, \& Dziuban 2000):

1. Phobic - Tend to fear negative outcomes, spending their energy in caution while taking care to consider every possible outcome. Highly analytical. 
2. Impulsive - Unpredictable and often energetic. These students tend to perform on a whim, frequently engaging in tasks without careful consideration or prior experience.

3. Obsessive Compulsive - Organized and methodical. These students tend to complete their tasks and be successful. While typically beneficial, their unspontaneous work ethic can be exhausting.

4. Hysterical-Creative, empathetic, and openly emotional. When feeling positive, their energy is contagious. However, they have an affinity for crisis, reacting in a strong negative manner if they perform poorly on a test or forget an assignment.

The Long-Dziuban Survey Instrument (Dziuban \& Dziuban, 1998) is a validated (Long, 1985) and reliable (Cioffi, 1995) instrument used to assess these types and traits. As a short two question self-report instrument, its utility partially lies in its simplicity. The first question asks the reader to read four short descriptions and mark the one that they most identify with, thereby indicating his or her type. The second question asks the reader to read four more short descriptions and mark as many as he or she identifies with, thereby indicating his or her traits. If Long's types are influential in the effectiveness of badges, the simplicity of this instrument will prove useful in efficiently deciding whether or not to use badges with specific groups of students.

Existing research supports the instrument's ability to differentiate between varying levels of student performance. For example, reactive behavior types and traits of students, as assigned by the survey instrument, have been influential in academic achievement in mathematics (Cioffi, 1995; Junkins, 2000), real estate examination performance (Combs, 2004), and student-desired instructor feedback methodology in dance education (Salapa, 2000). This study will focus on the interaction between these reactive behavior types and traits and a digital badging system in university-level courses. 


\section{RESEARCH QUESTIONS}

The primary research question inquires whether or not individual differences exist on the effectiveness of a digital distance-learning badging system with regard to reactive behavior types and traits. If so, more specific research questions include:

1. How does aggressiveness impact badging effectiveness?

2. How does dependence impact badging effectiveness?

3. How does reactive behavior type impact badging effectiveness?

4. How do LD traits impact badging effectiveness?

5. To what extent and in which direction does number of badges earned correlate with the various dimensions of badging effectiveness?

For this study, effectiveness is defined along several dimensions, including engagement, intrinsic motivation, and the seven dimensions measured by the National Survey for Student Engagement (NSSE): collaborative learning, reflective \& integrative learning, student faculty interaction, higher order learning, effective teaching practices, learning strategies, and student satisfaction.

\section{METHOD}

\section{Participants}

Participants in this study included 44 students ( 24 male, 20 female) from the University of Central Florida (UCF). All participants were over the age of 18 and currently enrolled in one of four web-based courses, two Web Design sections, and two Graphic Design sections. All course sections had the same instructor and were offered as electives for students in UCF's School of Visual Arts and Design.

In total, 89 students consented to participation in this study. However, all surveys were provided online. Several participants elected to not complete any of the surveys, or did not complete the entire set of surveys. Only complete datasets were retained, totaling 44 participants. No discernable patterns of differences were observed in the available data between complete and incomplete datasets. 
Participants were not offered any incentives or penalties for participating in the study.

\section{Course Structure}

Both courses were completely web-based with an equal emphasis on quizzes and exams and project-based assignments. The courses were structured to improve ability in web and graphic design while preparing students for the Adobe Certified Expert exams in either Dreamweaver or Photoshop. For Web Design, the course consisted of complementary projects in Dreamweaver that built into a complete website by the end of the semester. In Graphic Design, participants completed a series of projects in Photoshop, including digital image editing, video editing, and various aspects of design.

\section{Badging System}

Two of the sections, one for each course, included a badging system. Originally, the badging system contained a total of 22 badges (18 were present in Graphic Design, 19 in Web Design). Four badges were never awarded. Top Gun, So Close, and Third Wheel would have been awarded for the top three scores on each project. However, a clear top three never emerged as multiple participants consistently occupied the top three positions. Flawless victory would have been awarded for receiving a perfect score on all quizzes, a feat which was not accomplished by any participants. Thus, a total of 18 badges remained (14 in Graphic Design, 15 in Web Design). See Table 1. 
Table 1. Badges and Associated Criteria

\begin{tabular}{|c|c|c|}
\hline Badge Name & Criteria & Course \\
\hline Let's Play a Game & $\begin{array}{l}\text { Automatically awarded on } \\
\text { first day of class }\end{array}$ & Both Courses \\
\hline High Score & Score $100 \%$ on a project & Both Courses \\
\hline $\begin{array}{l}\text { Do or do not. There is no } \\
\text { try }\end{array}$ & $\begin{array}{l}\text { Score } 100 \% \text { on a quiz or } \\
\text { exam }\end{array}$ & Both Courses \\
\hline Pre-emptive Strike & $\begin{array}{l}\text { Finish an exam at least two } \\
\text { days before its due date and } \\
\text { score a } 90 \% \text { or higher }\end{array}$ & Both Courses \\
\hline $\begin{array}{l}\text { All your basics are } \\
\text { belong to us }\end{array}$ & $\begin{array}{l}\text { Score } 90 \% \text { or higher on all of } \\
\text { the first three quizzes }\end{array}$ & Both Courses \\
\hline $\begin{array}{l}\text { YOU SHALL NOT } \\
\text { PASS! }\end{array}$ & $\begin{array}{l}\text { Score } 90 \% \text { or higher on all } \\
\text { quizzes }\end{array}$ & Both Courses \\
\hline You... complete me & $\begin{array}{l}\text { Finish final project before the } \\
\text { last day it is due and score a } \\
90 \% \text { or better }\end{array}$ & Both Courses \\
\hline Above the call of duty & $\begin{array}{l}\text { Exceptional effort on a project } \\
\text { (as perceived by instructor) }\end{array}$ & Both Courses \\
\hline Happy Little Trees & $\begin{array}{l}\text { Exceptional creativity on a } \\
\text { project (as perceived by } \\
\text { instructor) }\end{array}$ & Both Courses \\
\hline Dr. Phil & $\begin{array}{l}\text { Provide high quality } \\
\text { constructive feedback on the } \\
\text { discussion board (as } \\
\text { perceived by instructor) }\end{array}$ & Both Courses \\
\hline Overachiever & $\begin{array}{l}\text { Effectively demonstrate an } \\
\text { advanced concept not covered } \\
\text { by the curriculum }\end{array}$ & Both Courses \\
\hline Well Planned & $\begin{array}{l}\text { Initial site skeleton is an } \\
\text { accurate representation of } \\
\text { final published site }\end{array}$ & Web Design Only \\
\hline Optimized Prime & $\begin{array}{l}\text { Site loads quickly with no } \\
\text { errors on a mobile device }\end{array}$ & Web Design Only \\
\hline
\end{tabular}




\begin{tabular}{lll}
\hline I have a dream & $\begin{array}{l}\text { First assignment site mockup } \\
\text { demonstrates exceptional } \\
\text { detail }\end{array}$ & Web Design Only \\
One site to rule them all & $\begin{array}{l}\text { No site errors on any of the } \\
\text { five tested browsers }\end{array}$ & Web Design Only \\
Too Easy & $\begin{array}{l}\text { Used more than the required } \\
\text { number of images in } \\
\text { assignment 7's composition }\end{array}$ & \\
Don't Ever Change & $\begin{array}{l}\text { Used adjustment layers in a } \\
\text { project }\end{array}$ & Graphic Design Only \\
I shall call him squishy & $\begin{array}{l}\text { Named all layers within a } \\
\text { project that included 3 or } \\
\text { more layers. }\end{array}$ & \\
& Graphic Design Only
\end{tabular}

Badges were introduced by the "Let's Play a Game" badge, which was awarded to all students on the first day of class. Subtext accompanying the badge indicated that many other badges were obtainable, but that students would not be told how to unlock them. In accordance with Blair (2012), aside from one introductory badge, all badges were skill-based, requiring the demonstration of advanced ability or exceptional effort to obtain the badges. While some were awarded for achieving a perfect score, others were given for reasons such as performing well and submitting an assignment early, demonstrating exceptional creativity, or helping another student on the discussion board. Examples of badge designs are included in Figure 1.
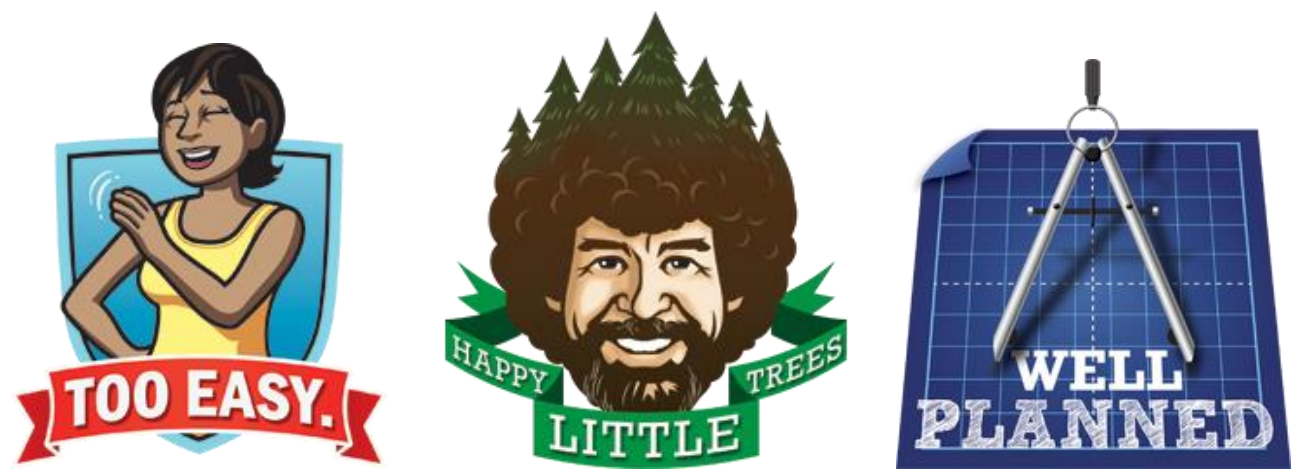

Fig 1. Badge Examples 
Participants could view their earned badges at any time by clicking a link labeled "Achievements" on the course website. Badges of other students were not visible. Badges not yet acquired were not shown or mentioned anywhere on the site. Clicking an earned badge revealed a description of the badge, the completion criterion, and a larger image of the badge.

\section{Materials}

To measure reactive behavior type and associated traits, the Long-Dziuban Survey Instrument was used. This measure asks participants to read four short descriptions and select the one that best matches them, thereby indicating their reactive behavior type. Next, they read a description of four traits and select any number of traits that they feel describes them.

A modified version of the NSSE (NSSE 2014) was used to assess collaborative learning, reflective and integrative learning, student faculty interaction, higher order learning, effective teaching practices, learning strategies, and student satisfaction. Modifications were made to better suit the structure of the course, removing responses that were irrelevant (e.g., asking if student gave a presentation in the course. This was not an option for this web-based course).

Other measures included the Interest/Enjoyment Subscale of the Intrinsic Motivation Inventory (Ryan, 1982) to measure intrinsic motivation, and the Engagement Measure (Charlton \& Danforth, 2005; Jennett et al., 2008) to measure engagement. Final grade and number of badges earned were also recorded.

\section{Procedure}

All participants proceeded through their respective course as any student would. Measures were disseminated online three weeks before the end of the semester to facilitate higher completion rates.

Students in badged courses were awarded badges both automatically and by the instructor, depending on the type of badge. Badges that were based on concrete criteria for a single project or exam were awarded automatically (e.g., received a $100 \%$ on an exam). Subjective badges were always awarded by the instructor (e.g., demonstrated exceptional creativity on a project). 
Students in non-badged courses proceeded through the course ordinarily, with the exception of completing the measures at the end of the semester. No badges were awarded in these courses

Completion of study materials was optional. No benefits were offered to students who participated. Students who did not participate did not experience any punishment and still received badges if they were in the badged courses.

\section{Data Analysis}

T-tests and correlation analyses were conducted with alpha defined at 0.05 . For correlations, a large effect is considered at $r>0.50$, medium at $r>0.30$. Effects below 0.30 were considered negligible and are not reported. T-tests were used for all significance testing.

\section{RESULTS}

During data collection, only a small number of participants classified themselves as not phobic in non-badge courses $(n=4)$, aggressive independent (AI) in either course type $\left(\mathrm{n}_{\text {badges }}=2, \mathrm{n}_{\text {noBadges }}=2\right)$, or passive independent $(\mathrm{PI})$ in non-badge courses $(n=4)$. To reduce the potential for confounds, analyses that examined these groups were not conducted. However, AI and PI participants were included when examining independent types in general. The total participant breakdown can be seen in Table 2 and Table 3 .

Table 2. Participant Breakdown By Long Type

\begin{tabular}{llllll}
\hline & AI & PI & AD & PD & Total \\
\hline Badged & 2 & 4 & 10 & 5 & 21 \\
Non-Badged & 2 & 6 & 9 & 6 & 23 \\
Total & 4 & 10 & 19 & 11 & 44 \\
\hline
\end{tabular}


Table 3. Participant Breakdown by Long Trait

\begin{tabular}{llllll}
\hline & Phobic & $\begin{array}{l}\text { Obsessive } \\
\text { Compulsive }\end{array}$ & Impulsive & Hysteric & None \\
\hline Badged & 13 & 14 & 5 & 6 & 3 \\
Non-Badged & 19 & 14 & 5 & 10 & 0 \\
Total & 32 & 28 & 10 & 16 & 3 \\
\hline
\end{tabular}

Minimum and maximum observed and possible values for each construct are listed in Table 4. Note that lower values are more desirable than higher values for NSSE items (collaborative learning, reflective and integrative learning, student faculty interaction, higher order learning, effective teaching practices, learning strategies, and student satisfaction). A higher score for these items represents a lower score on the construct. Also note that Pearson's r values have been converted for easier interpretation so that a positive value reflects a positive relationship with the construct. No other conversions have occurred.

Table 4. Minimum and Maximum Values by Construct

\begin{tabular}{lllllll}
\hline & $\begin{array}{l}\text { Minimum } \\
\text { Observed }\end{array}$ & $\begin{array}{l}\text { Maximum } \\
\text { Observed }\end{array}$ & $\begin{array}{l}\text { Absolute } \\
\text { Minimum }\end{array}$ & $\begin{array}{l}\text { Absolute } \\
\text { Maximum }\end{array}$ & Mean & SD \\
\hline Final Grade & 63.90 & 98.20 & 0 & 100 & 86.35 & 8.77 \\
$\begin{array}{l}\text { Intrinsic } \\
\text { Motivation }\end{array}$ & 10 & 47 & 7 & 49 & 37.30 & 9.31 \\
$\begin{array}{l}\text { Engagement } \\
\text { Collaborative }\end{array}$ & 30 & 60 & 12 & 60 & 47.59 & 8.03 \\
$\begin{array}{l}\text { Learning } \\
\text { R\&I Learning }\end{array}$ & 3 & 12 & 3 & 12 & 10.02 & 2.33 \\
$\begin{array}{l}\text { Student Faculty } \\
\text { Interaction }\end{array}$ & 7 & 9 & 3 & 12 & 5.66 & 1.77 \\
$\begin{array}{l}\text { Higher Order } \\
\text { Learning }\end{array}$ & 4 & 16 & 4 & 16 & 13.95 & 2.39 \\
$\begin{array}{l}\text { Effective } \\
\text { Teaching Practices }\end{array}$ & 5 & 18 & 4 & 20 & 9.80 & 3.59 \\
$\begin{array}{l}\text { Learning } \\
\text { Strategies }\end{array}$ & 2 & 17 & 5 & 25 & 9.93 & 3.45 \\
$\begin{array}{l}\text { Student } \\
\text { Satisfaction }\end{array}$ & 2 & 7 & 2 & 8 & 4.18 & 1.76 \\
\hline & & & 2 & & & \\
\hline
\end{tabular}




\section{Research Question 1 Results - Aggressive vs Passive Types}

In badge courses, aggressive types scored significantly better than passive types in intrinsic motivation, $\mathrm{t}(19)=-2.90 ; \mathrm{p}=0.01$, engagement, $\mathrm{t}(19)=-2.95 ; \mathrm{p}=0.01$, reflective and integrative learning, $\mathrm{t}(19)=2.43 ; \mathrm{p}=0.03$, and higher order learning, $\mathrm{t}(19)=4.03 ; \mathrm{p}<0.01$. See Table 5, Table 6, and Table 7.

Table 5. Means and SDs for Aggressive and Passive Types in Badge Courses

\begin{tabular}{lllll}
\hline & $\mathrm{M}_{\text {aggressive }}$ & $\mathrm{SD}_{\text {aggressive }}$ & $\mathrm{M}_{\text {passive }}$ & $\mathrm{SD}_{\text {passive }}$ \\
\hline Final Grade & 86.24 & 6.81 & 85.04 & 13.03 \\
Intrinsic Motivation & 41.58 & 4.60 & 32.00 & 10.24 \\
Engagement & 51.00 & 5.69 & 42.22 & 7.97 \\
Collaborative Learning & 9.75 & 2.56 & 11.11 & 0.78 \\
R\&I Learning & 5.08 & 1.83 & 7.00 & 1.73 \\
Student Faculty & 12.67 & 3.09 & 14.00 & 2.96 \\
Interaction & 7.92 & 2.75 & 12.44 & 2.24 \\
Higher Order Learning & 9.42 & 3.48 & 11.11 & 2.85 \\
Effective Teaching & 3.92 & 1.73 & 5.11 & 1.05 \\
Practices & 3.17 & 1.19 & 4.22 & 1.39 \\
Learning Strategies & Student Satisfaction & & &
\end{tabular}


Table 6. Means and SDs for Aggressive and Passive Types in Non-badged Courses

\begin{tabular}{lllll}
\hline & $\mathrm{M}_{\text {aggressive }}$ & $\mathrm{SD}_{\text {aggressive }}$ & $\mathrm{M}_{\text {passive }}$ & $\mathrm{SD}_{\text {passive }}$ \\
\hline Final Grade & 87.16 & 7.45 & 86.71 & 8.83 \\
Intrinsic Motivation & 38.82 & 8.09 & 35.58 & 11.56 \\
Engagement & 50.00 & 6.56 & 46.00 & 9.47 \\
Collaborative Learning & 9.55 & 2.16 & 9.92 & 2.94 \\
R\&I Learning & 5.00 & 1.10 & 5.83 & 1.80 \\
Student Faculty & 14.64 & 1.43 & 14.58 & 1.38 \\
Interaction & 8.36 & 3.14 & 11.00 & 4.13 \\
Higher Order Learning & 9.36 & 3.93 & 10.08 & 3.55 \\
Effective Teaching & 3.45 & 1.51 & 4.42 & 2.19 \\
Practices & 3.55 & 1.44 & 4.17 & 1.80 \\
Learning Strategies & & & & \\
Student Satisfaction & & &
\end{tabular}

Table 7. p-values for Research Question 1: Aggressive and Passive Types

\begin{tabular}{|c|c|c|c|c|}
\hline & $\begin{array}{l}\text { Agg. vs Pass. } \\
\text { Badge } \\
\text { Courses } \\
\end{array}$ & $\begin{array}{l}\text { Agg. vs Pass. } \\
\text { Non-Badge } \\
\text { Courses }\end{array}$ & $\begin{array}{l}\text { Agg. } \\
\text { Badges vs } \\
\text { No Badges } \\
\end{array}$ & $\begin{array}{l}\text { Pass. } \\
\text { Badges vs } \\
\text { No Badges } \\
\end{array}$ \\
\hline Final Grade & 0.79 & 0.90 & 0.76 & 0.73 \\
\hline Intrinsic Motivation & $* * 0.01$ & 0.45 & 0.32 & 0.47 \\
\hline Engagement & $* * 0.01$ & 0.26 & 0.70 & 0.35 \\
\hline $\begin{array}{l}\text { Collaborative } \\
\text { Learning }\end{array}$ & 0.14 & 0.74 & 0.84 & 0.25 \\
\hline R\&I Learning & $* * 0.03$ & 0.20 & 0.90 & 0.15 \\
\hline $\begin{array}{l}\text { Student Faculty } \\
\text { Interaction }\end{array}$ & 0.33 & 0.93 & 0.07 & 0.55 \\
\hline $\begin{array}{l}\text { Higher Order } \\
\text { Learning }\end{array}$ & $* *<0.001$ & 0.10 & 0.72 & 0.36 \\
\hline $\begin{array}{l}\text { Effective Teaching } \\
\text { Practices }\end{array}$ & 0.25 & 0.65 & 0.97 & 0.49 \\
\hline Learning Strategies & 0.08 & 0.24 & 0.50 & 0.39 \\
\hline Student Satisfaction & 0.07 & 0.37 & 0.50 & 0.94 \\
\hline
\end{tabular}




\section{Research Question 2 Results - Independent vs Dependent Types}

Dependent types had significantly higher student faculty interaction, $\mathrm{t}(28)=2.06$; $\mathrm{p}=0.05$, in badge courses than non-badge courses.

In non-badge courses, independent types had significantly lower engagement than dependent types, $\mathrm{t}(21)=3.55 ; \mathrm{p}<0.01$. See Table 8, Table 9, and Table 10 .

Table 8. Means and SDs. for Independent and Dependent Types in Badged Courses

\begin{tabular}{lllll}
\hline & $\mathrm{M}_{\text {independent }}$ & $\mathrm{SD}_{\text {independent }}$ & $\mathrm{M}_{\text {dependent }}$ & $\mathrm{SD}_{\text {dependent }}$ \\
\hline Final Grade & 84.38 & 11.72 & 82.27 & 9.16 \\
Intrinsic Motivation & 38.50 & 8.02 & 37.07 & 9.31 \\
Engagement & 48.67 & 8.76 & 46.67 & 7.84 \\
Collaborative Learning & 10.67 & 1.03 & 10.20 & 2.40 \\
R\&I Learning & 5.33 & 1.63 & 6.13 & 3.13 \\
Student Faculty & 13.17 & 3.82 & 13.27 & 2.82 \\
Interaction & 9.50 & 4.68 & 10.00 & 2.90 \\
Higher Order Learning & 8.83 & 4.17 & 10.67 & 2.82 \\
Effective Teaching & 4.50 & 1.64 & 4.40 & 1.60 \\
Practices & 3.33 & 1.21 & 3.73 & 1.44 \\
Learning Strategies & & & & \\
Student Satisfaction & & &
\end{tabular}


Table 9. Means and SDs. for Independent and Dependent Types in Non-badged Courses

\begin{tabular}{lllll}
\hline & $\mathrm{M}_{\text {independent }}$ & $\mathrm{SD}_{\text {independent }}$ & $\mathrm{M}_{\text {dependent }}$ & $\mathrm{SD}_{\text {dependent }}$ \\
\hline Final Grade & 85.59 & 9.37 & 87.64 & 7.46 \\
Intrinsic Motivation & 32.13 & 9.27 & 39.80 & 9.54 \\
Engagement & 41.13 & 6.92 & 51.53 & 6.59 \\
Collaborative Learning & 10.63 & 2.13 & 9.27 & 2.69 \\
R\&I Learning & 5.75 & 2.19 & 5.27 & 1.10 \\
Student Faculty & 14.00 & 1.20 & 14.93 & 1.39 \\
Interaction & 10.75 & 5.04 & 9.20 & 3.12 \\
Higher Order Learning & 9.88 & 3.40 & 9.67 & 3.92 \\
Effective Teaching & 4.38 & 2.39 & 3.37 & 1.67 \\
Practices & 4.75 & 1.58 & 3.40 & 1.50 \\
Learning Strategies & & & & \\
Student Satisfaction & 4.53 & & \\
\hline
\end{tabular}

Table 10. p-values for Research Question 2: Independent and Dependent Types

\begin{tabular}{lllll}
\hline & $\begin{array}{l}\text { Indep vs } \\
\text { Dep Badge } \\
\text { Courses }\end{array}$ & $\begin{array}{l}\text { Indep vs Dep } \\
\text { Non-Badge } \\
\text { Courses }\end{array}$ & $\begin{array}{l}\text { Badges vs } \\
\text { No Badges } \\
\text { Indep }\end{array}$ & $\begin{array}{l}\text { Badges vs } \\
\text { No Badges } \\
\text { Dep }\end{array}$ \\
\hline Final Grade & 0.70 & 0.57 & 0.83 & 0.66 \\
Intrinsic Motivation & 0.75 & 0.08 & 0.20 & 0.43 \\
Engagement & 0.62 & $* *<0.001$ & 0.10 & 0.08 \\
Collaborative Learning & 0.65 & 0.23 & 0.33 & 0.32 \\
R\&I Learning & 0.42 & 0.48 & $* * 0.02$ & 0.17 \\
$\begin{array}{l}\text { Student Faculty } \\
\text { Interaction }\end{array}$ & 0.95 & 0.12 & 0.55 & $* * 0.05$ \\
Higher Order Learning & 0.77 & 0.37 & 0.15 & 0.47 \\
$\begin{array}{l}\text { Effective Teaching } \\
\text { Practices }\end{array}$ & 0.25 & 0.90 & 0.35 & 0.43 \\
Learning Strategies & 0.90 & 0.46 & 0.53 & 0.27 \\
$\begin{array}{l}\text { Student Satisfaction } \\
\text { **significant (p<.05) }\end{array}$ & 0.40 & 0.06 & 0.53 & 0.54 \\
\hline
\end{tabular}




\section{Research Question 3 Results - Individual Types}

Aggressive Dependent and Passive Dependent Types

Passive dependent (PD) types had significantly lower reflective and integrative learning in badge courses than non-badge courses, $t(9)=-3.00 ; p=0.02$. In badge courses, Aggressive Dependent (AD) types had significantly higher intrinsic motivation, $\mathrm{t}(13)=2.31 ; \mathrm{p}=0.04$, engagement, $\mathrm{t}(13)=2.60 ; \mathrm{p}=0.02$, reflective and integrative learning, $\mathrm{t}(13)=-3.01 ; \mathrm{p}=0.01$, and higher order learning, $\mathrm{t}(13)=$ $-3.13 ; \mathrm{p}=0.01$, than PDs. See Table 11, Table 12, and Table 13.

Table 11. Means and SDs. for AD and PD Types in Badged Courses

\begin{tabular}{lllll}
\hline & $\mathrm{M}_{\mathrm{AD}}$ & $\mathrm{SD}_{\mathrm{AD}}$ & $\mathrm{M}_{\mathrm{PD}}$ & $\mathrm{SD}_{\mathrm{PD}}$ \\
\hline Final Grade & 85.74 & 7.22 & 87.32 & 13.19 \\
Intrinsic Motivation & 40.50 & 4.25 & 30.20 & 13.20 \\
Engagement & 49.80 & 5.45 & 40.40 & 8.62 \\
Collaborative Learning & 9.70 & 2.79 & 11.20 & 0.84 \\
R\&I Learning & 5.20 & 1.87 & 8.00 & 1.23 \\
Student Faculty & 12.60 & 3.10 & 14.60 & 1.67 \\
Interaction & 8.70 & 2.26 & 12.60 & 2.30 \\
Higher Order Learning & 10.30 & 3.09 & 11.40 & 2.30 \\
Effective Teaching & 4.10 & 1.79 & 5.00 & 1.00 \\
Practices & 3.40 & 1.17 & 4.40 & 1.82 \\
Learning Strategies & Student Satisfaction & & & \\
\hline
\end{tabular}


Table 12. Means and SDs. for AD and PD Types in Non-badged Courses

\begin{tabular}{lllll}
\hline & $\mathrm{M}_{\mathrm{AD}}$ & $\mathrm{SD}_{\mathrm{AD}}$ & $\mathrm{M}_{\mathrm{PD}}$ & $\mathrm{SD} \mathrm{PD}$ \\
\hline Final Grade & 86.63 & 8.20 & 89.15 & 6.60 \\
Intrinsic Motivation & 41.56 & 5.81 & 37.17 & 13.67 \\
Engagement & 52.22 & 4.79 & 50.50 & 9.09 \\
Collaborative Learning & 9.11 & 2.15 & 9.50 & 3.56 \\
R\&I Learning & 4.89 & 0.93 & 5.83 & 1.17 \\
Student Faculty & 14.78 & 1.48 & 15.17 & 1.33 \\
Interaction & 8.78 & 3.11 & 9.83 & 3.31 \\
Higher Order Learning & 9.78 & 4.27 & 9.50 & 3.73 \\
Effective Teaching & 3.33 & 1.32 & 4.33 & 2.07 \\
Practices & 3.22 & 1.30 & 3.67 & 1.86 \\
Learning Strategies & & &
\end{tabular}

Table 13. p-values for Research Question 3: Individual Types - AD and PD

\begin{tabular}{lllll}
\hline & $\begin{array}{l}\text { AD vs } \\
\text { PD } \\
\text { Badge } \\
\text { Courses }\end{array}$ & $\begin{array}{l}\text { AD vs PD } \\
\text { Courses }\end{array}$ & $\begin{array}{l}\text { Badges vs } \\
\text { No Badges } \\
\text { AD }\end{array}$ & $\begin{array}{l}\text { Badges vs } \\
\text { No Badges } \\
\text { PD }\end{array}$ \\
\hline Final Grade & 0.77 & 0.54 & 0.80 & 0.77 \\
Intrinsic Motivation & $* * 0.04$ & 0.40 & 0.66 & 0.42 \\
Engagement & $* * 0.02$ & 0.64 & 0.32 & 0.09 \\
Collaborative Learning & 0.27 & 0.80 & 0.62 & 0.33 \\
R\&I Learning & $* * 0.01$ & 0.11 & 0.66 & $* * 0.02$ \\
$\begin{array}{l}\text { Student Faculty } \\
\text { Interaction }\end{array}$ & 0.21 & 0.61 & 0.07 & 0.55 \\
Higher Order Learning & $* * 0.01$ & 0.54 & 0.95 & 0.15 \\
$\begin{array}{l}\text { Effective Teaching } \\
\text { Practices }\end{array}$ & 0.5 & 0.90 & 0.76 & 0.35 \\
Learning Strategies & 0.32 & 0.27 & 0.31 & 0.53 \\
$\begin{array}{l}\text { Student Satisfaction } \\
\text { **significant (p <.05) }\end{array}$ & 0.22 & 0.59 & 0.76 & 0.53 \\
\hline
\end{tabular}




\section{Research Question 4 Results- Individual Traits}

\section{Phobic}

In badge courses, non-phobic participants scored significantly better on reflective and integrative learning, $\mathrm{t}(19)=-2.99 ; \mathrm{p}=0.01$, and higher order learning, $\mathrm{t}(19)=$ $-3.19 ; \mathrm{p}=0.01$, than phobic participants. See Table 14 and Table 15.

Table 14. Means and SDs. for Phobic and Non-phobic Traits in Badged Courses

\begin{tabular}{lllll}
\hline & $\mathrm{M}_{\text {phobic }}$ & $\mathrm{SD}_{\text {phobic }}$ & $\mathrm{M}_{\text {nonPhobic }}$ & $\mathrm{SD}_{\text {nonPhobic }}$ \\
\hline Final Grade & 85.28 & 11.65 & 86.45 & 5.85 \\
Intrinsic Motivation & 35.69 & 10.18 & 40.38 & 5.24 \\
Engagement & 44.85 & 8.75 & 51.13 & 4.55 \\
Collaborative Learning & 10.23 & 2.35 & 10.50 & 1.69 \\
R\&I Learning & 6.77 & 1.69 & 4.50 & 1.69 \\
$\begin{array}{l}\text { Student Faculty } \\
\text { Interaction }\end{array}$ & 14.00 & 2.92 & 12.00 & 2.98 \\
Higher Order Learning & 11.38 & 2.60 & 7.38 & 3.11 \\
$\begin{array}{l}\text { Effective Teaching } \\
\text { Practices }\end{array}$ & 10.23 & 2.56 & 10.00 & 4.38 \\
Learning Strategies & 4.77 & 1.59 & 3.88 & 1.46 \\
Student Satisfaction & 3.92 & 1.50 & 3.13 & 0.99
\end{tabular}


Table 15. p-values for Research Question 4: Individual Traits - Phobic and Not Phobic

\begin{tabular}{lllll}
\hline & $\begin{array}{l}\text { Phobic vs } \\
\text { Not Phobic } \\
\text { Badge } \\
\text { Courses }\end{array}$ & $\begin{array}{l}\text { Phobic vs } \\
\text { Not Phobic } \\
\text { Non-Badge } \\
\text { Courses }\end{array}$ & $\begin{array}{l}\text { Badges vs } \\
\text { No Badges } \\
\text { Phobic }\end{array}$ & $\begin{array}{l}\text { Badges vs } \\
\text { No Badges } \\
\text { Not Phobic }\end{array}$ \\
\hline Final Grade & 0.80 & --- & 0.71 & --- \\
Intrinsic Motivation & 0.25 & --- & 0.81 & --- \\
Engagement & 0.08 & --- & 0.46 & --- \\
Collaborative Learning & 0.78 & --- & 0.56 & --- \\
R\&I Learning & $* * 0.01$ & --- & 0.06 & --- \\
$\begin{array}{l}\text { Student Faculty } \\
\text { Interaction }\end{array}$ & 0.15 & --- & 0.34 & --- \\
Higher Order Learning & $* * 0.01$ & --- & 0.32 & --- \\
Effective Teaching & 0.88 & --- & 0.91 & --- \\
$\begin{array}{l}\text { Practices } \\
\text { Learning Strategies }\end{array}$ & 0.21 & --- & 0.17 & --- \\
Student Satisfaction & 0.20 & --- & 0.83 & --- \\
\hline **significant (p<.05) & & & &
\end{tabular}

Obsessive Compulsive

No significant results were observed for obsessive compulsive participants. See

Table 16, Table 17, and Table 18. 
Table 16. Means and SDs. for Obsessive Compulsive and Non-obsessive Compulsive Traits in Badged Courses

\begin{tabular}{lllll}
\hline & $\mathrm{M}_{\mathrm{OC}}$ & $\mathrm{SD}_{\mathrm{OC}}$ & $\mathrm{M}_{\text {nonOC }}$ & $\mathrm{SD}_{\text {nonOC }}$ \\
\hline Final Grade & 85.46 & 8.13 & 86.26 & 13.00 \\
Intrinsic Motivation & 39.00 & 7.21 & 34.43 & 11.33 \\
Engagement & 47.50 & 7.52 & 46.71 & 9.32 \\
Collaborative Learning & 10.36 & 2.31 & 10.29 & 1.70 \\
R\&I Learning & 5.86 & 1.79 & 6.00 & 2.52 \\
Student Faculty & 13.29 & 2.87 & 13.14 & 3.58 \\
Interaction & 9.86 & 3.26 & 9.86 & 3.89 \\
Higher Order Learning & 9.64 & 2.90 & 11.14 & 3.93 \\
Effective Teaching & 4.36 & 1.69 & 4.57 & 1.40 \\
Practices & 3.57 & 1.16 & 3.71 & 1.80 \\
Learning Strategies & Student Satisfaction & & & \\
\hline
\end{tabular}

Table 17. Means and SDs. for Obsessive Compulsive and Non-obsessive Compulsive Traits in Non-badged Courses

\begin{tabular}{lllll}
\hline & $\mathrm{M}_{\mathrm{OC}}$ & $\mathrm{SD}_{\mathrm{OC}}$ & $\mathrm{M}_{\text {nonOC }}$ & $\mathrm{SD}_{\text {nonOC }}$ \\
\hline Final Grade & 86.19 & 7.77 & 88.08 & 8.74 \\
Intrinsic Motivation & 34.50 & 11.54 & 41.22 & 5.04 \\
Engagement & 45.43 & 8.54 & 51.78 & 6.52 \\
Collaborative Learning & 9.50 & 2.71 & 10.11 & 2.37 \\
R\&I Learning & 5.43 & 1.70 & 5.44 & 1.33 \\
Student Faculty & 14.64 & 1.45 & 14.56 & 1.33 \\
Interaction & 10.14 & 4.40 & 9.11 & 2.93 \\
Higher Order Learning & 10.57 & 3.76 & 8.44 & 3.32 \\
Effective Teaching & 4.29 & 2.27 & 3.44 & 1.13 \\
Practices & 4.00 & 1.75 & 3.67 & 1.50 \\
Learning Strategies & & &
\end{tabular}


Table 18. p-values for Research Question 4: Individual Traits - Obsessive Compulsive and Not Obsessive Compulsive

\begin{tabular}{|c|c|c|c|c|}
\hline & $\begin{array}{l}\text { Obs Comp vs } \\
\text { Not Obs } \\
\text { Comp Badge } \\
\text { Courses }\end{array}$ & $\begin{array}{l}\text { Obs Comp vs } \\
\text { Not Obs } \\
\text { Comp } \\
\text { Non-Badge } \\
\text { Courses }\end{array}$ & $\begin{array}{l}\text { Badges vs } \\
\text { No Badges } \\
\text { Obs Comp }\end{array}$ & $\begin{array}{l}\text { Badges vs } \\
\text { No Badges } \\
\text { Not Obs } \\
\text { Comp }\end{array}$ \\
\hline Final Grade & 0.87 & 0.59 & 0.81 & 0.74 \\
\hline Intrinsic Motivation & 0.27 & 0.12 & 0.23 & 0.13 \\
\hline Engagement & 0.83 & 0.07 & 0.50 & 0.22 \\
\hline Collaborative Learning & 0.94 & 0.59 & 0.38 & 0.87 \\
\hline R\&I Learning & 0.88 & 0.98 & 0.52 & 0.58 \\
\hline $\begin{array}{l}\text { Student Faculty } \\
\text { Interaction }\end{array}$ & 0.92 & 0.89 & 0.13 & 0.29 \\
\hline Higher Order Learning & 1.00 & 0.54 & 0.85 & 0.67 \\
\hline $\begin{array}{l}\text { Effective Teaching } \\
\text { Practices }\end{array}$ & 0.33 & 0.18 & 0.47 & 0.16 \\
\hline Learning Strategies & 0.78 & 0.32 & 0.93 & 0.10 \\
\hline Student Satisfaction & 0.83 & 0.64 & 0.45 & 0.96 \\
\hline
\end{tabular}

\section{Impulsive}

Impulsive participants had significantly lower learning strategies, $\mathrm{t}(8)=-2.80 ; \mathrm{p}=$ 0.02 , in badge courses than non-badge courses. Non-impulsive participants had significantly higher student faculty interaction, $\mathrm{t}(32)=2.33 ; \mathrm{p}=0.03$, in badge courses than non-badge courses. In badge courses, non-impulsive participants scored significantly better than impulsive participants in intrinsic motivation, $t(19)$ $=2.11 ; \mathrm{p}=0.05$, engagement, $\mathrm{t}(19)=3.35 ; \mathrm{p}<0.01$, reflective $\&$ integrative learning, $\mathrm{t}(19)=-2.07 ; \mathrm{p}=0.05$, and learning strategies, $\mathrm{t}(19)=-3.06 ; \mathrm{p}=0.01$. See Table 19, Table 20, and Table 21. 
Table 19. Means and SDs. for Impulsive and Non-Impulsive Traits in Badged Courses

\begin{tabular}{lllll}
\hline & $\mathrm{M}_{\mathrm{imp}}$ & $\mathrm{SD}_{\text {imp }}$ & $\mathrm{M}_{\text {nonImp }}$ & $\mathrm{SD}_{\text {nonImp }}$ \\
\hline Final Grade & 85.98 & 11.28 & 85.65 & 9.54 \\
Intrinsic Motivation & 30.80 & 12.19 & 39.56 & 6.61 \\
Engagement & 38.80 & 7.79 & 49.87 & 6.05 \\
Collaborative Learning & 10.00 & 3.39 & 10.44 & 1.63 \\
R\&I Learning & 7.40 & 1.52 & 5.44 & 1.93 \\
Student Faculty & 14.20 & 3.49 & 12.94 & 2.93 \\
Interaction & 11.60 & 2.51 & 9.31 & 3.50 \\
Higher Order Learning & 10.80 & 1.64 & 9.94 & 3.64 \\
Effective Teaching & 6.00 & 1.23 & 3.94 & 1.34 \\
Practices & 4.60 & 1.95 & 3.31 & 1.01 \\
Learning Strategies & & &
\end{tabular}

Table 20. Means and SDs. for Impulsive and Non-Impulsive Traits in Non-badged Courses

\begin{tabular}{lllll}
\hline & $\mathrm{M}_{\mathrm{imp}}$ & $\mathrm{SD}_{\text {imp }}$ & $\mathrm{M}_{\text {nonImp }}$ & $\mathrm{SD}_{\text {nonImp }}$ \\
\hline Final Grade & 82.74 & 6.18 & 88.09 & 8.23 \\
Intrinsic Motivation & 35.20 & 9.12 & 37.67 & 10.36 \\
Engagement & 43.40 & 6.19 & 49.17 & 8.49 \\
Collaborative Learning & 10.60 & 2.61 & 9.50 & 2.55 \\
R\&I Learning & 6.20 & 2.28 & 5.22 & 1.26 \\
Student Faculty & 14.20 & 1.64 & 14.72 & 1.32 \\
Interaction & 9.80 & 4.82 & 9.72 & 3.71 \\
Higher Order Learning & 8.00 & 2.55 & 10.22 & 3.84 \\
Effective Teaching & 3.40 & 1.67 & 4.11 & 2.00 \\
Practices & 4.60 & 1.95 & 3.67 & 1.53 \\
Learning Strategies & & & & \\
Student Satisfaction & & &
\end{tabular}


Table 21. p-values for Research Question 4: Individual Traits - Impulsive and Not Impulsive

\begin{tabular}{|c|c|c|c|c|}
\hline & $\begin{array}{l}\text { Impulsive } \\
\text { vs } \\
\text { Not } \\
\text { Impulsive } \\
\text { Badge } \\
\text { Courses }\end{array}$ & $\begin{array}{l}\text { Impulsive vs } \\
\text { Not Impulsive } \\
\text { Non-Badge } \\
\text { Courses }\end{array}$ & $\begin{array}{l}\text { Badges vs } \\
\text { No Badges } \\
\text { Impulsive }\end{array}$ & $\begin{array}{l}\text { Badges vs } \\
\text { No Badges } \\
\text { Not } \\
\text { Impulsive }\end{array}$ \\
\hline Final Grade & 0.95 & 0.19 & 0.56 & 0.43 \\
\hline Intrinsic Motivation & $* * 0.05$ & 0.64 & 0.54 & 0.54 \\
\hline Engagement & $* *<0.001$ & 0.17 & 0.33 & 0.78 \\
\hline Collaborative Learning & 0.69 & 0.41 & 0.76 & 0.22 \\
\hline R\&I Learning & $* * 0.05$ & 0.21 & 0.36 & 0.70 \\
\hline $\begin{array}{l}\text { Student Faculty } \\
\text { Interaction }\end{array}$ & 0.43 & 0.47 & 1.00 & $* * 0.03$ \\
\hline Higher Order Learning & 0.19 & 0.97 & 0.48 & 0.74 \\
\hline $\begin{array}{l}\text { Effective Teaching } \\
\text { Practices }\end{array}$ & 0.62 & 0.24 & 0.07 & 0.83 \\
\hline Learning Strategies & $* * 0.01$ & 0.48 & $* * 0.02$ & 0.77 \\
\hline Student Satisfaction & 0.06 & 0.27 & 1.00 & 0.44 \\
\hline
\end{tabular}

Hysteric

No significant results were observed for hysteric participants. See Table 22, Table 23, and Table 24. 
Table 22. Means and SDs. for Hysteric and Non-hysteric Traits in Badged Courses

\begin{tabular}{lllll}
\hline & $\mathrm{M}_{\text {hyst }}$ & $\mathrm{SD}_{\text {hyst }}$ & $\mathrm{M}_{\text {nonHyst }}$ & $\mathrm{SD}_{\text {nonHyst }}$ \\
\hline Final Grade & 84.23 & 7.83 & 86.33 & 10.52 \\
Intrinsic Motivation & 35.33 & 8.57 & 38.33 & 9.01 \\
Engagement & 43.83 & 7.31 & 48.60 & 8.00 \\
Collaborative Learning & 11.50 & 0.84 & 9.87 & 2.26 \\
R\&I Learning & 5.67 & 2.16 & 6.00 & 2.00 \\
$\begin{array}{l}\text { Student Faculty } \\
\text { Interaction }\end{array}$ & 14.00 & 1.67 & 12.93 & 3.43 \\
Higher Order Learning & 9.83 & 3.06 & 9.87 & 3.60 \\
$\begin{array}{l}\text { Effective Teaching } \\
\text { Practices }\end{array}$ & 10.83 & 2.71 & 9.87 & 3.50 \\
Learning Strategies & 4.50 & 1.87 & 4.40 & 1.50 \\
Student Satisfaction & 3.83 & 0.75 & 3.53 & 1.55 \\
\hline
\end{tabular}

Table 23. Means and SDs. for Hysteric and Non-hysteric Traits in Non-badged Courses

\begin{tabular}{lllll}
\hline & $\mathrm{M}_{\text {hyst }}$ & $\mathrm{SD}_{\text {hyst }}$ & $\mathrm{M}_{\text {nonHyst }}$ & $\mathrm{SD}_{\text {nonHyst }}$ \\
\hline Final Grade & 87.07 & 8.68 & 86.82 & 7.83 \\
Intrinsic Motivation & 40.90 & 5.38 & 34.23 & 11.80 \\
Engagement & 49.60 & 7.38 & 46.62 & 8.98 \\
Collaborative Learning & 9.30 & 3.13 & 10.08 & 2.06 \\
R\&I Learning & 5.40 & 1.35 & 5.46 & 1.71 \\
Student Faculty & 14.10 & 1.37 & 15.00 & 1.29 \\
Interaction & 9.80 & 3.52 & 9.69 & 4.23 \\
Higher Order Learning & 8.70 & 2.95 & 10.54 & 4.08 \\
Effective Teaching & 3.20 & 1.23 & 4.54 & 2.18 \\
Practices & 3.80 & 1.55 & 3.92 & 1.75 \\
Learning Strategies & & & & \\
Student Satisfaction & 3.54 & & \\
\hline
\end{tabular}


Table 24. p-values for Research Question 4: Individual Traits - Hysteric and Not Hysteric

\begin{tabular}{lllll}
\hline & $\begin{array}{l}\text { Hysteric vs } \\
\text { Not Hysteric } \\
\text { Badge } \\
\text { Courses }\end{array}$ & $\begin{array}{l}\text { Hysteric vs } \\
\text { Not Hysteric } \\
\text { Non-Badge } \\
\text { Courses }\end{array}$ & $\begin{array}{l}\text { Badges vs } \\
\text { No Badges } \\
\text { Hysteric }\end{array}$ & $\begin{array}{l}\text { Badges vs } \\
\text { No Badges } \\
\text { Not Hysteric }\end{array}$ \\
\hline Final Grade & 0.67 & 0.94 & 0.52 & 0.89 \\
Intrinsic Motivation & 0.49 & 0.11 & 0.13 & 0.31 \\
Engagement & 0.22 & 0.40 & 0.15 & 0.54 \\
Collaborative Learning & 0.11 & 0.48 & 0.12 & 0.80 \\
R\&I Learning & 0.74 & 0.93 & 0.76 & 0.46 \\
$\begin{array}{l}\text { Student Faculty } \\
\text { Interaction }\end{array}$ & 0.48 & 0.12 & 0.90 & 0.05 \\
$\begin{array}{l}\text { Higher Order Learning } \\
\text { Effective Teaching }\end{array}$ & 0.98 & 0.95 & 0.99 & 0.91 \\
$\begin{array}{l}\text { Practices } \\
\text { Learning Strategies }\end{array}$ & 0.55 & 0.24 & 0.17 & 0.64 \\
$\begin{array}{l}\text { Student Satisfaction } \\
\text { **significant (p<.05) }\end{array}$ & 0.66 & 0.86 & 0.96 & 0.54 \\
\hline
\end{tabular}

\section{Research Question 5 - Correlations}

The correlations between all variables and number of badges earned are listed in Table 25 and Table 26. 
Table 25. Aggressiveness \& Dependence Correlations

\begin{tabular}{lllllll}
\hline & AD & PD & Indep. & Dep. & Agg. & Passive \\
\hline $\begin{array}{l}\text { Student } \\
\text { Satisfaction }\end{array}$ & -.301 & -.905 & -.305 & $* *-.557$ & -.253 & $* *-.685$ \\
$\begin{array}{l}\text { Learning } \\
\text { Strategies }\end{array}$ & -.173 & -.339 & -.758 & -.197 & -.265 & -.429 \\
$\begin{array}{l}\text { Effective } \\
\text { Teaching }\end{array}$ & .113 & -.125 & -.387 & .035 & .06 & -.14 \\
$\begin{array}{l}\text { Practices } \\
\text { Higher Order }\end{array}$ & .236 & $* *-.979$ & -.128 & -.174 & .134 & -.364 \\
$\begin{array}{l}\text { Learning } \\
\text { Student }\end{array}$ & & & & & & \\
$\begin{array}{l}\text { Faculty } \\
\text { Interaction }\end{array}$ & .177 & .122 & -.471 & .136 & -.047 & .136 \\
$\begin{array}{l}\text { Reflective \& } \\
\text { Integrative }\end{array}$ & .037 & -.484 & $* *-.904$ & -.091 & -.136 & -.211 \\
$\begin{array}{l}\text { Learning } \\
\text { Collaborative }\end{array}$ & -.037 & .385 & $* *-.983$ & .015 & -.108 & .022 \\
$\begin{array}{l}\text { Learning } \\
\text { Engagement }\end{array}$ & -.076 & .321 & .464 & .104 & -.007 & .233 \\
$\begin{array}{l}\text { Intrinsic } \\
\text { Motivation }\end{array}$ & -.272 & .442 & .546 & .156 & -.182 & .407 \\
Final Grade & $* * .723$ & .861 & .506 & $* * .778$ & $* * .724$ & $* * .725$ \\
\hline$* *$ significant $(\mathrm{p}<.05)$ & & & & & & \\
\hline
\end{tabular}




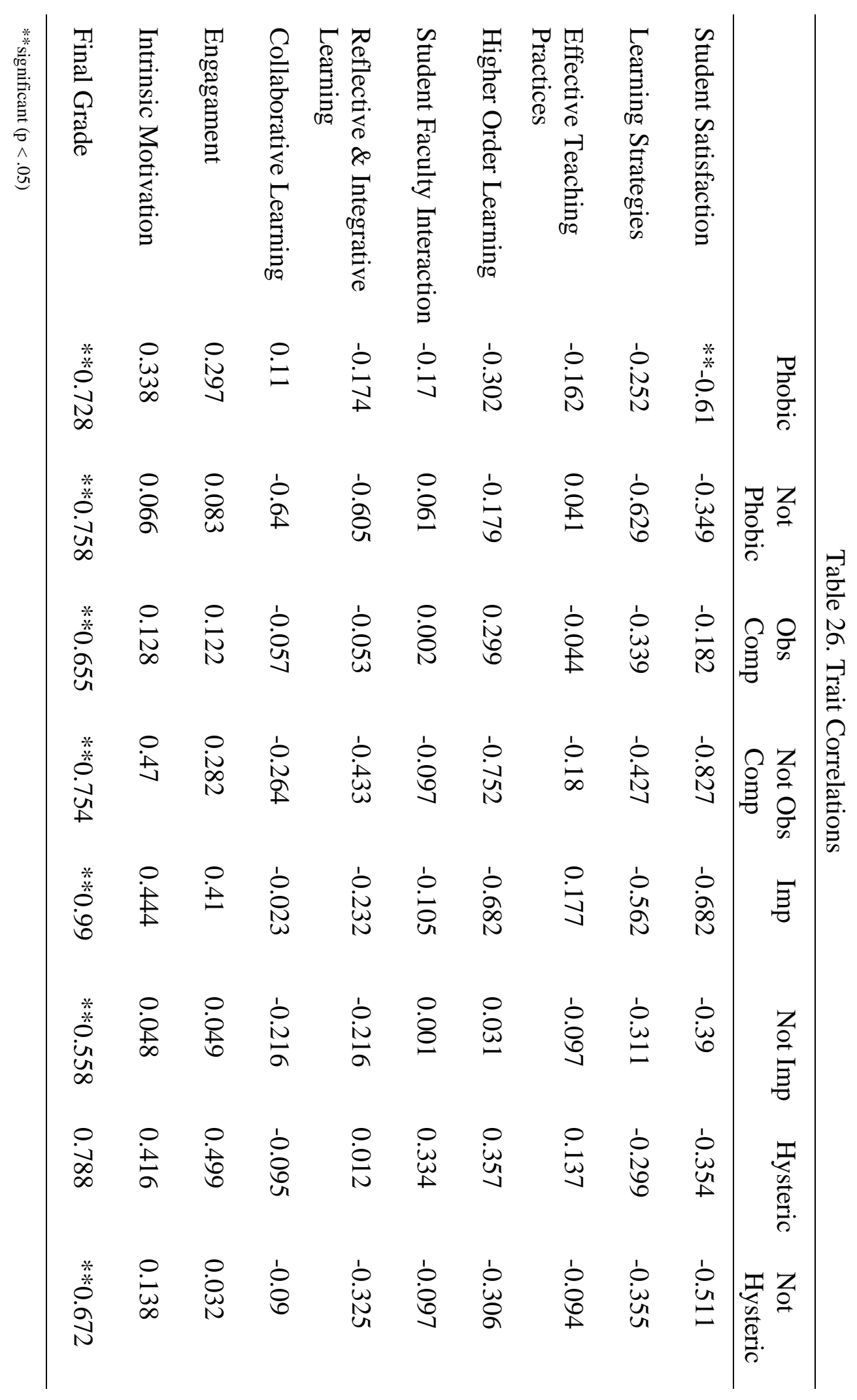




\section{DISCUSSION}

\section{Research Question 1 Discussion - Aggressive vs Passive Types}

Several differences were observed between aggressive and passive participants. First, aggressive participants consistently performed better in badged courses than passive participants, achieving significantly better scores in higher order learning, reflective and integrative learning, engagement, and intrinsic motivation. Additionally, aggressive participants had significantly better student faculty interaction in badge courses than non-badge courses. One may consider the possibility that passive participants did not have adequate drive to actively engage in the hunt for badges. If this were the case, it would be expected that passive participants would have received fewer badges than aggressive participants, which was not the case. Instead, the difference may be a reaction to other positive feedback received in response to additional work put in by aggressive participants (e.g., grades, instructor praise, etc.), with badges playing only a supplementary role. Further research should be conducted to gain a better grasp on the underlying causes for the observed effects.

\section{Research Question 2 Discussion - Independent vs Dependent Types}

Significant differences were sparse between independent and dependent participants. Dependent participants showed significantly higher engagement, but no other significant differences were observed between the two types.

Differences were also minimal between badge and non-badge participants within each type. Dependent participants had significantly higher student faculty interaction in badged courses than non-badged courses, while independent participants showed no significant differences between the two course types. The defining characteristic of dependents is that they require more approval than independents. Badges may seem like a great means of providing this approval, but they did not appear to adequately serve this role in this study. If badged courses increased perception of approval, dependents would likely have shown improved satisfaction in badge courses, probably accompanied by engagement. While dependents did show significantly higher engagement than independents, the 
effect was not seen between badge and non-badge courses within the dependent group. It may be proposed that while receiving a badge was likely viewed as a positive event which conveyed approval, submitting an assignment and not receiving a badge for it could have been perceived as the opposite. Instead of being perceived as the mere absence of approval, it may have delivered the message that the assignment submission was insufficient to be awarded a badge (approval), thus discouraging dependent students. Further, student faculty interaction was significantly higher in badge courses, which may have been a result of student attempts to resolve this inner conflict by gaining counsel from the professor. Future research should re-examine these groups with a badge system that has skill-based badges available for all assignments to see if the consistent potential for approval improves dependent outcomes. If increasing the number and consistency of badges increases outcomes for dependent students, these modifications are expected to have widespread beneficial effects.

\section{Research Question 3 Discussion - Individual Types}

Dependents in this study were further categorized into ADs and PDs. ADs consistently outperformed PDs in badged courses, scoring significantly better in intrinsic motivation, engagement, reflective and integrative learning, and higher order learning, while no significant differences between types were observed in non-badged courses.

PDs performed significantly worse in badge courses than non-badge courses for engagement and reflective and integrative learning. ADs suffered no similar ill effects. Given these results, it seems as though the positive effects described for all dependents, in research question 2, was primarily applied to PDs. While both ADs and PDs have a high need for approval, it seems likely that the higher energy levels from ADs resulted in higher performance, resulting in greater levels of positive feedback. PDs may not have had that experience and were unable to counteract the effects of not receiving badges for all assignments. Deeper insight into the effects observed for these two groups would be acquired through conducting research on badging systems that provide the opportunity to earn badges along regular intervals. 


\section{Research Question 4 Discussion - Individual Traits}

Obsessive compulsive and hysteric participants revealed no significant results. However, impulsive and phobic participants did show differences in comparison to their counterparts and the presence or non-presence of badges.

While there were no significant differences between impulsive and nonimpulsive participants in non-badged courses, non-impulsive participants showed improved scores in three dimensions in badged courses, including intrinsic motivation, engagement, and learning strategies. Additionally, non-impulsive participants scored significantly better on student faculty interaction in badged courses than non-badged courses, and impulsive students performed significantly worse in learning strategies in badged courses than non-badged courses. While badges seem to have several clear benefits to non-impulsive participants over impulsive participants, the cause is uncertain. Future studies should examine the underlying mechanisms for these results.

While there was an insufficient number of phobic participants to run analyses in non-badged courses, differences were found between phobic and nonphobic participants in badged courses, with non-phobics performing significantly better in higher order learning and, most interestingly, reflective and integrative learning. This is particularly interesting because phobics are defined by their analytic tendencies and careful considerations of possible outcomes. Despite this, phobics in badged courses performed less reflective and integrative learning than non-phobics. Looking deeper, the difference between reflective and integrative learning in phobics in non-badged courses and badged courses approached significance at $\mathrm{p}=0.06$. Badging may have had a negative impact on this type of learning for phobics, but the reason remains unclear. Larger scale studies should be conducted to more deeply examine the impact of badging systems on phobics and their counterparts. 


\section{Research Question 5 Discussion - Correlations}

Several relationships were observed, but a few, in particular, are highly remarkable. Student satisfaction showed a significant large positive relationship with number of badges earned, showing an increase in satisfaction as participants earned more badges. This evidence extends the theory posited earlier in this article, that dependents may have seen the absence of badges as proof of insufficiency, not merely the absence of approval. This support provides further testament to the need for future research examining the effects of badging technologies with more badges available along consistent intervals on dependent type learners. Additionally, independents showed medium or better positive relationships for number of badges earned with nearly all of the tested variables. Thus, it is unlikely that a negative effect would be seen for independents if more badges were included.

It was also proposed that the increased energy levels of AD participants may have resulted in better quality work, and thus higher levels of approval than their lower energy PD counterparts, protecting them from the negative effects on perception of approval from not receiving badges. The correlational data for PDs seems to fit, showing medium or better positive relationships between number of badges earned with final grade, intrinsic motivation, engagement, collaborative learning, reflective and integrative learning, higher order learning, learning strategies, and student satisfaction. As number of badges earned increased, so did these factors. Future research should undergo further examination of approval as a potential mediating factor.

\section{CONCLUSION}

While the majority of badge research has been conducted without regard for individual differences, the findings from this study are indicative of the importance of these factors. This study adds to the short but growing list of evidence for the existence of individual differences in the way students are affected by badges, while providing specific points of focus for future research.

In particular, passive dependent learners warrant increased attention. They did not benefit to the same level as aggressive dependent or independent learners. 
In response to the observed data, it is proposed that dependent learners will benefit from badging systems that offer badges more frequently, during regular intervals, to provide a more consistent source of potential approval. While this type of design is not expected to negatively impact non-passive dependent learners, they should also be included in future experimentation to ensure negative effects are not introduced.

Impulsiveness and Phobia were also impactful in this study, with badging showing reduced scores on several dimensions for impulsive and phobic participants over their counterparts. If a large percentage of learners for a specific course come from a population that is expected to have a high proportion of impulsiveness or phobia, a badging system may be undesirable. Future research should examine the underlying factors for this effect to see if it can be mitigated through careful design.

As badge prevalence continues to escalate, increasing importance will be placed on the knowledge of how students of varying characteristics react to various badging system designs. If optimization of a badging system is desirable and it is - badge studies should begin identifying the best implementation methodologies for learners of varying characteristics. Given the differences observed in this study, the Long-Dziuban instrument may be an effective starting point. Future research should take care to examine a range of learning styles and learner characteristics, including and beyond those mentioned here.

\section{REFERENCES}

Abramovich, S.J., Schunn, C., \& Higashi, R.M. (2013). Are badges useful in education?: It depends upon the type of badge and expertise of learner. Education Technology Research and Development, 61, 213-232.

Blair, L. (2012). The use of video game achievements to enhance player performance, self-efficacy, and motivation. Doctoral Dissertation. University of Central Florida, 1-30.

Carey, K. (2012). Show me your badge. The New York Times. http://www.nytimes.com/2012/11/04/education/edlife/show-me-yourbadge.html?pagewanted=all\&_r=0 
Charleer, S., Klerkx, J., Santos, J.L., \& Duval, E. (2013). Improving awareness and reflection through collaborative, interactive visualizations of badges. Proceedings of ARTEL '13, 69-81.

Charlton, J., \& Danforth, I. (2005). Distinguishing addiciton and high engagement in the context of online game playing. Computers in Human Behavior, 23(3), 1531-1548.

Combs, D. (2004). Predicting Licensing Examination Performance with Cognitive Style and Reactive Behavior Pattern Assessment (Doctoral Dissertation). Retrieved from ProQuest, UMI Dissertations Publishing (3162088).

Denny, P. (2013). The effect of virtual achievements on student engagement. Proceedings of CHI 2013, 763-772.

Dziuban, J.I. \& Dziuban, C.D. (1998). Reactive behavior patterns in the classroom. Journal of Staff, Program, and Organization Development, 15(2), 85-91.

Dziuban, C.D., Moskal, P.D., \& Dziuban, E.K. (2000). Reactive behavior patterns go online. Journal of Staff, Program \& Organization Development, 17(3), 171-182.

Fitzwalter, Z., Tjondronegoro, D., \& Wyeth, P. (2011). Orientation passport: Using gamification to engage university students. Proceedings of the $23^{\text {rd }}$ Australian Computer-Human Interaction Conference, 122-125.

Frederiksen, L. (2013). Digital badges. Public Services Quarterly, 9, 321-325.

Hakulinen, L. \& Auvinen, T. (2014). The effect of gamification on students with different achievement goal orientation. Proceedings of the International Conference on Teaching and Learning in Computing and Engineering, 916.

Jennett, C., Cox, A., Cairns, P., Dhoparee, S., Epps, A., Tijs, T., et al. (2008). Measuring and defining the experience of immersion in games. International Journal of Human Computer Studies, 66(9), 641-661.

Junkins, N.R. (2000). A study of the impact of long reactive behavior patterns on grade nine placement and academic achievement in mathematics (Doctoral Dissertation). Retrieved from ProQuest, UMI Dissertations Publishing (9977816).

Khaddage, F., Baker, R., \& Knezek, G. (2012). If not now! When? A mobile 
badge reward system for K-12 teachers. Proceedings of the Society for Information Technology \& Teacher Education International Conference, 2900-2905.

Long, W.A. (1975). Adolescent maturation: A clinical overview. Postgraduate Medicine, 57(3), 54-60.

Long, W.A.. (1985). The practitioner and adolescent medicine. Seminars in Adolescent Medicine, 1(1), 85-90.

Mehta, N.B., Hull, A.L., Young, J.B., \& Stoller, J.K. (2013). Just imagine: New paradigms for medical education. Academic Medicine, 88(10), 1418-1423.

Mozilla (2014). Mozilla OpenBadges. http://www.openbadges.org

NSSE (2014). Survey Instrument. National Survey For Student Engagement. Retrieved from http://nsse.iub.edu/html/survey_instruments.cfm

Purdue (2014). Passport. Purdue University. http://www.itap.purdue.edu/studio/passport/

Rosewell, J. (2012). A speculation on the possible use of badges for learning at the UK Open University. Procedings of the EADTU Annual Conference: The Role of Open and Flexible Education in European Higher Education Systems for 2020: New Models, New Markets, New Media, 1-15.

Ryan, R. M. (1982). Control and Information in the intrapersonal sphere: An extension of cognitive evaluation theory. Journal of Personality and Social Psychology, 43, 450-461.

Salapa, S. (2000). The Relationship between Student Personality Types and Traits and Instructor Feedback in Dance Education. (Unpublished Doctoral Dissertation). University of Central Florida, Orlando.

Stewart, M. (2013). Digital badges at the agricultural sustainability institute. The Wheel: The Instructional Technology Blog of ATS at UC Davis. http://wheel.ucdavis.edu/2013/11/digital-badges-at-the-agriculturalsustainability-institute/ 